\title{
Role of Oxidative Stress and the Microenvironment in Breast Cancer Development and Progression
}

\author{
Agnieszka Jezierska-Drutel ${ }^{\star}$, Steven A. Rosenzweig ${ }^{*}$, and Carola A. Neumann ${ }^{\star}, t, 1$ \\ "Department of Cell and Molecular Pharmacology and Experimental Therapeutics, Medical \\ University of South Carolina, Charleston, South Carolina, USA \\ tDepartment of Pharmacology and Chemical Biology, University of Pittsburgh Cancer Institute, \\ Magee-Womens-Research Institute, Pittsburgh, Pennsylvania, USA
}

\section{Abstract}

Breast cancer is a highly complex tissue composed of neoplastic and stromal cells. Carcinomaassociated fibroblasts (CAFs) are commonly found in the cancer stroma, where they promote tumor growth and enhance vascularity in the microenvironment. Upon exposure to oxidative stress, fibroblasts undergo activation to become myofibroblasts. These cells are highly mobile and contractile and often express numerous mesenchymal markers. CAF activation is irreversible, making them incapable of being removed by nemosis. In breast cancer, almost $80 \%$ of stromal fibroblasts acquire an activated phenotype that manifests by secretion of elevated levels of growth factors, cytokines, and metalloproteinases. They also produce hydrogen peroxide, which induces the generation of subsequent sets of activated fibroblasts and tumorigenic alterations in epithelial cells. While under oxidative stress, the tumor stroma releases high energy nutrients that fuel cancer cells and facilitate their growth and survival. This review describes how breast cancer progression is dependent upon oxidative stress activated stroma and proposes potential new therapeutic avenues.

\section{Keywords}

Oxidative stress; Reactive oxygen species; Breast cancer; Microenvironment; Stroma-associated fibroblasts; Myofibroblasts; Cancer-associated fibroblasts

\section{OXIDATIVE STRESS CHANGES THE BREAST TUMOR MICROENVIRONMENT}

\footnotetext{
Cancer is one of the most common diseases in the United States (Siegel, Naishadham, \& Jemal, 2012). In 2012, breast cancer alone was expected to account for $29 \%(226,870)$ of all new cancer cases among women in the United States. It is known that one in eight women will develop breast cancer during their lives, and almost 40,000 will die because of breast cancer metastasis. Men are also at risk of breast cancer and it is estimated that in 2012, almost 2190 men will be diagnosed with breast cancer, and 410 will die due to this disease. Breast cancer is not only an isolated group of mutated somatic cells, but it is also a microenvironment system comprising breast cancer cells, fibroblasts, adipocytes, immune, and endothelial cells (Fig. 3.1). Close interactions between cancer cells and stroma are known to regulate breast cancer pathways. Among the many factors influencing
}

\footnotetext{
(c) 2013 Elsevier Inc. All rights reserved.

${ }^{1}$ Corresponding author: neumannc@upmc.edu.
} 
development, progression, and metastasis, oxidative stress has an important role in the initiation and preservation of breast cancer progression. Oxidative stress results from an imbalance between unstable reactive species lacking one or more unpaired electrons (superoxide anion, hydrogen peroxide, hydroxyl radical, reactive nitrogen species) and antioxidants. Oxidative stress can be generated by ultraviolet light exposure, ionizing radiation, or carcinogen exposure. One way in which cellular reactive oxygen species (ROS) impact cell signaling is through their localized accumulation, if we consider ROS byproducts of the electron transport chain in mitochondria or activation of the NADPH oxidases (NOX). Induced pathways producing ROS function in all cell types, including both breast carcinoma and cancer stroma (Hecker et al., 2009; Sampson et al., 2011; Tobar et al., 2010). Stromal-derived NOX4 ROS are able to stimulate migration of MCF-7 cells in a paracrine manner (Tobar et al., 2010). Overexpression of NOX4 in normal breast epithelial cells results in cellular senescence, resistance to apoptosis, and tumorigenic transformation, as well as increased aggressiveness of breast cancer cells (Graham et al., 2010).

Interestingly, several recent studies have confirmed that ROS, including hydrogen peroxide, alone are able to drive the differentiation of normal fibroblasts into myofibroblasts, which are able to generate high amounts of hydrogen peroxide themselves, increasing oxidative stress in the microenvironment (Comito et al., 2012; Taddei et al., 2012; Toullec et al., 2010; Waghray et al., 2005) (Fig. 3.2). Almost $80 \%$ of fibroblasts acquire an activated phenotype in breast cancer (Kalluri \& Zeisberg, 2006; Sappino et al., 1988). Myofibroblasts are highly mobile and contractile cells that typically express mesenchymal markers such as a-smooth muscle actin (a-SMA), calponin, and vimentin (Kalluri \& Zeisberg, 2006). Additionally, type I collagen secreted by myofibroblasts causes higher breast density, which contributes to mammary tumor formation and metastasis (Provenzano et al., 2008). It has also been shown that type I collagen contributes to decreased chemotherapeutic agent uptake and altered tumor cell sensitivity to a variety of chemotherapies. Not only collagen deposition but also collagen degradation is required for the physiologic remodeling of connective tissue during breast cancer growth, development, and cancer cell invasion. Secreted metalloproteinases (MMP) such as MMP-2, MMP-3, and MMP-9 increase extracellular matrix turnover and are themselves activated by oxidative stress (Fu et al., 2001; Koch et al., 2009; Provenzano et al., 2008; Radisky et al., 2005). Additionally secreted transforming growth factor $\beta$ (TGF $\beta$ ), insulin-like growth factor (IGF), platelet-derived growth factor (PDGF), fibroblast growth factor 2, and stromal-derived factor 1 (SDF1) are able to activate fibroblasts and increase cancer cell proliferation (Sappino et al., 1988). Growth factors such as TGF $\beta$, IGF, TNFa, or PDGF stimulate ROS production through NOX (Basuroy et al., 2009; Edderkaoui et al., 2011; Marumo et al., 1997; Meng, Lv, \& Fang, 2008). Once activated, the tumor microenvironment network produces large amounts of ROS, initiating tumor growth. It would be clinically advantageous to detect this large ROS activity early enough to prevent further cancer progression. There is only one clinical study showing that such a possibility exists. The research involving an equal number of breast cancer patients and healthy controls showed increased levels of hydrogen peroxide in exhaled breath condensate from patients with localized breast malignancy, associated with increased clinical severity (Stolarek et al., 2010).

\section{OXIDATIVE STRESS REGULATE CAVEOLIN-1 SIGNALING AND ENERGY METABOLISM IN THE TUMOR MICROENVIRONMENT}

Since 1920, the "Warburg Effect" has been the leading principle in understanding breast cancer development resulting from dysfunctional mitochondrial oxidative phosphorylation (Koppenol, Bounds, \& Dang, 2011). This mechanism was shown to be incomplete. Specifically based on the "Warburg Effect," the recently proposed "Reverse Warburg Effect" underscores an important and supportive role for oxidative stress and the 
microenvironment in cancer progression (Balliet et al., 2011). It was shown that aerobic glycolysis occurs in the adjacent stroma, rather than in cancer cells. Stromal autophagy drives cancer cell growth and progression by providing fatty acids, nucleotides, and free amino acids that are ready for use by cancer cells. Moreover, catabolites produced by stroma-associated fibro-blasts not only stimulate mitochondrial biogenesis in breast cancer cells, they also protect them from autophagy and chemotherapy-induced apoptosis (Martinez-Outschoorn et al., 2010a, 2011a, 2013). Comparing mitochondrial metabolic activity revealed a difference between stroma and epithelial cells (Balliet et al., 2011). While breast cancer epithelial cells appear to have increased mitochondrial oxidative activity, as shown by their elevated levels of NADH dehydrogenase, saccharopine dehydrogenase (SDH), and cyclooxygenase (Whitaker-Menezes et al., 2011a). Activated stroma fibroblasts, on the other hand, exhibit a low mitochondrial oxidative capacity, low translocase of outer mitochondrial membrane 20, yet high expression of monocarboxylate transporter 4 (MCT4) and the lysosomal protease-cathepsin B. Not surprisingly, breast cancer tumors that exhibit upregulation of enzymes involved in oxidative phosphorylation are prone to metastasize to the brain, which itself is a lactate-rich environment (Sonveaux et al., 2008; Sotgia, MartinezOutschoorn, \& Lisanti, 2011; Sotgia et al., 2012). This creates an ideal condition for tumor progression. A recent study has shown that cancer cells are able to induce drivers of oxidative stress, autophagy and mitophagy: HIF-1a and NFkB in surrounding stroma fibroblasts (Martinez-Outschoorn et al., 2011b). This process can lead to loss of stromal caveolin-1 (Cav-1) (Martinez-Outschoorn et al., 2010b). Cav-1 is highly expressed in differentiated fibroblasts, adipocytes, and endothelial cells (Lisanti et al., 1994). Loss of Cav-1 in CAFs is consideNorthwestern University Feinberg School of Medicine, Chicago, IL, USAred to be a "lethal" breast tumor microenvironment, as it is linked with tamoxifen resistance, increased lymph node metastasis, early tumor recurrence, and poor overall outcome (Martinez-Outschoorn et al., 2010c; Witkiewicz et al., 2011). In triple negative breast cancer stroma, the loss of Cav-1 in $\sim 10 \%$ of cases was linked with a $\mathbf{5}$-year survival rate compared to approximately $75 \%$ of cases where Cav-1 stroma-positive patients had a 12 year survival rate (Witkiewicz et al., 2010). Interestingly, oxidative stress is sufficient to induce the loss of Cav-1 in fibroblasts due to lysosomal degradation of Cav-1 (MartinezOutschoorn et al., 2010a, 2010c). In support of this, knockdown of Cav-1 in immortalized human fibroblasts (hTERT-BJ1) results in increased hydrogen peroxide generation, which can further induce Cav-1 loss in neighboring fibroblasts (Trimmer et al., 2011). Studies show that loss of Cav-1 in adjacent breast cancer stroma fibroblasts can be prevented by treatment with $\mathrm{N}$-acetyl cysteine, quercetin, or metformin (Martinez-Outschoorn et al., 2010c; Witkiewicz et al., 2011). It was reported that overexpression of recombinant (SOD2) (Trimmer et al., 2011) or injection of SOD, catalase, or their pegylated counterparts can block recurrence and metastasis in mice (Goh et al., 2011; Hyoudou et al., 2008; Nishikawa et al., 2005). Of note, mammary stromal cells fromCav-1 (-/-) null mice have upregulated myofibroblast markers (vimentin, calponin2, tropomyosin, gelsolin, and prolyl 4hydroxylase alpha), EF-1- $\delta$ (elongation factor 1-delta: which can drive cell transformation and tumorigenesis), signaling molecules (annexin A1, annexin A2, and RhoGDI), glycolytic enzymes (M2-isoform of pyruvate kinase-PKM2 and LDHA), secreted proteins (type I collagen and SPARC), and two peroxidases: catalase and peroxiredoxin-1 (Prdx1) (Pavlides et al., 2009). Consistent with this premise, a study exam-ining mammary stroma from human breast cancer specimens using laser-capture microdissection and molecular profiling revealed that patients with Cav-1 negative breast cancer stroma had higher expression of genes associ-ated with oxidative stress, hypoxia, redox signaling, apoptosis, autophagy, lysosomal degradation, glycolysis aging, "stemness," inflammation, DNA damage, and myofibroblast differentiation (Witkiewicz et al., 2011). The reduction of Cav-1 occurs in both subtypes of breast cancers: estrogen receptor positive $\mathrm{ER}(+)$, and estrogen receptor negative ER(-). However, it was shown to be significantly associated with ER(-) cancers, 
increased recurrence, and decreased overall survival (Witkiewicz et al., 2011), suggesting perhaps that hormone receptor negative breast cancers are linked to higher levels of ROS. Along those lines, when exposed to estrogenic polychlorinated biphenyls, the triple negative breast cancer cell line MDA-MB-231 produced significantly higher amounts of ROS than the poorly metastatic T47D cell line (Lin \& Lin, 2006). Moreover, treatment of breast cancer cells with physiologic levels of 17 beta estradiol induces ROS production and activates redox sensitive signaling pathways in neighboring cells, leading to angiogenesis, tumor growth, and invasiveness (Felty, 2011). Lowered expression of Cav-1 not only leads to myofibroblast conversion and inflammation but also seems to impact aerobic glycolysis, leading to secretion of high energy metabolites such as pyruvate and lactate that drive mitochondrial oxidative phosphorylation in cancer cells (Balliet et al., 2011). Pyruvate dehydrogenase, the enzyme that converts pyruvate into acetyl-coA, is decreased in stromal fibroblasts due to Cav-1 loss (Martinez-Outschoorn et al., 2010c). Depletion of Cav-1 activates HIF-1a and creates a lactate-rich environment due to the forced conversion of aerobic pyruvate into lactate (Sonveaux et al., 2008; Sotgia et al., 2011b). It is widely held that HIF-1a function is dependent upon its location within the tumor microenvironment. It acts as a tumor promoter in CAFs and as a tumor suppressor in cancer cells (Chiavarina et al., 2010). Oxidative stress generated by breast cancer cells activates HIF-1a and NFKB in fibroblasts, leading to autophagy and lysosomal degradation of Cav-1 (Martinez-Outschoorn et al., 2010c). Thus, the level of Cav-1 in the microenvironment serves as an ideal marker for oxidative stress, hypoxia, and autophagy. The close relationship between oxidative stress, Cav-1, and energy metabolism is further illustrated by the finding that loss of Cav-1 results in the expression of two monocarboxylate transporters: MCT1 and MCT4. MCT4 expression is only increased in breast cancer-associated fibroblasts, whereas MCT1 expression appears to be restricted to breast cancer cells. As this allows for proper import of lactate from fibroblasts into cancer cells (Sonveaux et al., 2008; Whitaker-Menezes et al., 2011b), such findings again underscore the intimate relationship between these two compartments. In support of this, higher levels of MCT4 in the tumor stroma are associate with poor outcome in triple negative breast cancer (Sonveaux et al., 2008; Witkiewicz et al., 2012), while MCT4 expression in breast cancer cells had no significant predictive value (Sonveaux et al., 2008; Whitaker-Menezes et al., 2011b). In contrast, MCT1 expression in breast cancer cells was linked to p53 mutations, p53 loss, and poor outcome (Boidot et al., 2012). Given that the prognostic value of MCT4 is restricted to breast cancer stroma and MCT1 to breast cancer epithelial cells, MCT4, and MCT1 in combination with Cav-1, may be functional markers of oxidative stress and aerobic glycolysis, exclusively in tumor stroma.

\section{OXIDATIVE STRESS CAUSES FIBROBLASTS TO UNDERGO SENESCENCE AND INDUCES MITOCHONDRIAL DYSFUNCTION}

By the age of 40, breast cancer risk in women is 1 in 203, but it rapidly increases: for women over 40 years of age, it is 1 in 28 and by age 70, 1 in 15 females will develop breast cancer. Over the years, somatic mutations accumulate and increase the risk of developing breast cancer. Phenotypically, senescent cells partially resemble aging cells. For example, senescent fibroblasts are locked in a nonproliferative state, suffer multiple phenotypic changes and are able to secrete factors influencing endothelial cells, pushing them toward a carcinogenic pathway (Parrinello et al., 2005). Besides other stressors, including ionizing radiation, oncogenes, and environmental factors, hydrogen peroxide is one of the main factors that can push fibroblasts and cancer cells into senescence (Capparelli et al., 2012a). It was reported that hydrogen peroxide- induced senescence and autophagy are closely linked processes that may be mutually induced (Capparelli et al., 2012a). Cyclin-dependent kinase (CDK) is a crucial factor in initiating DNA synthesis during the mammalian cell division 
cycle. CDKs are regulated by CDK inhibitors such as p16 and p19, which also function as tumor suppressors (Bardeesy et al., 2006; Capparelli et al., 2012a; Sharpless et al., 2004; Weitzman, 2001). In CAFs, the CDK inhibitors p19 and smARF drive senescence and autophagy, resulting in increased angiogenesis and promotion of tumor growth. Fibroblasts expressing cell cycle inhibitors, such as p19 and smARF, are predisposed to hydrogen peroxide initiated senescence. In human ductal breast carcinoma in situ (DCIS), increases in p16 are linked with a ninefold increased risk of recurrence (Capparelli et al., 2012a). Recent data have revealed that cell cycle arrest, senescence, and autophagy are closely related biological processes, which can be induced by the same stimuli and can arise simultaneously in a given cell. Senescence can induce autophagy in CAFs and they undergo senescenceautophagy transition (Capparelli et al., 2012a). It was shown that overexpression of autophagy inducers in fibroblasts leads to autophagy. Moreover, these fibroblasts express the active senescence markers $\beta$-galactosidase and p21 and they gain a senescenceassociated secretory phenotype resulting in secretion of numerous growth factors, cytokines, and proteases that promote migration and invasion of cancer cells, independent of angiogenesis (Capparelli et al., 2012b, 2012c; Coppe et al., 2010). Of note, selective induction of autophagy only in breast cancer cells inhibits tumor growth. Fibroblast senescence is able to attenuate the functional differentiation of cells and increases branching morphogenesis of normal mammary epithelial cells (Krtolica et al., 2001; Parrinello et al., 2005). Branching is a physiological process in which secretion of soluble factors such as hepatocyte growth factor (HGF), MMP2, and MMP3 by fibroblasts, induces epithelial cells to invade and migrate through collagen in a controlled manner. Therefore, during carcinogenesis, fibroblasts support branching but control of this process is lost (Parrinello et al., 2005). Moreover, in aging mammary glands, the number of senescent fibroblasts along with IL-6 and -8 secretion increases. This in turn promotes malignant processes such as epithelial-mesenchymal transition (EMT) in premalignant epithelial cells (Campisi, 2013).

Recent studies show that in the breast cancer microenvironment, oxidative stress causes mitochondrial dysfunction, which manifests itself by the upregulation of numerous factors, such as the nuclear respiratory factor 1 (NRF1), which was shown to be upregulated in cancer stroma due to oxidative stress (Witkiewicz et al., 2011). NRF1 is a transcription factor that activates the expression of genes regulating cellular growth, respiration, and mitochondrial DNA (mtDNA) transcription and replication (Witkiewicz et al., 2011). Hydrogen peroxide was shown to increase levels of mtDNA absence sensitive factorGOLPH3/MIDAS. This oncogene modulates mTOR signaling and is able to increase mitochondrial mass by regulating mitochondrial lipids in response to mitochondrial dysfunction (Witkiewicz et al., 2011). mtDNA is extremely sensitive to oxidative damage due to the lack of protective histones and efficient repair mechanisms located in the nucleus. This is the mechanism by which excess mitochondrial ROS promotes malignant cell transformation. It was recently demonstrated that transgenic mice expressing the human catalase gene (mCAT) in mammary tumors had reduced primary tumor invasiveness and decreased pulmonary metastatic tumor incidence compared to mice that lacked mCAT (Goh et al., 2011). The presence of mCAT in fibroblasts leads to altered phosphorylation of p38MAPK (downstream ROS signaling pathway) in response to hydrogen peroxide (Goh et al., 2011). This lowered oxidative stress and selectin expression and decreased the colonization ability and metastatic potential of cancer cells. Mitochondrial malfunction leads to oversaturation with ROS in cancer cells and thereby cancer risk. This detrimental process is comprised of numerous factors. For example, loss of mitochondrial transcription factor A results in compromised transcription of mtDNA (Balliet et al., 2011). Persistent exposure to oxidative stressors or other DNA damaging agents eventually leads to accumulation of DNA mutations in every tissue. Therefore, increased cancer risk with aging can be attributed to somatic mutations or alterations of gene copy number in the adjacent stroma. Particularly in breast cancer, somatic mutations in TP53 and PTEN and gene copy number alterations at 
other loci in the adjacent stroma have been described (Haviv et al., 2009). PTEN, a protein tyrosine and lipid phosphatase, is a crucial tumor suppressor in most cancers, including breast. PTEN belongs to a class of phosphatases known to be prone to inactivation induced by oxidative stress. Supporting this premise, we have recently shown that Prdx1 prevents PTEN oxidation-induced inactivation preventing $\mathrm{H}$-Ras and human epidermal growth factor 2 (ErbB-2)-driven transformations (Cao et al., 2009). Over the last few years, an impressive body of work describing PTEN's tumor suppressive role in epithelial cells, including breast, has accumulated (Gonzalez-Angulo et al., 2011; Heering, Erlmann, \& Olayioye, 2009; Jensen et al., 2012; Noh et al., 2011; Russillo et al., 2011; Suda et al., 2012; Tanic et al., 2012). However, a very recent elegant study defined PTEN's tumor suppressive role in mammary stromaassociated fibroblasts (Trimboli et al., 2009), enforcing the importance of oxidative stress promoting cancer through regulating the tumor microenvironment. mtDNA polymorphisms and oxidative stress sensitivity may be underappreciated factors in breast carcinogenesis. The mtDNA 10398A allele was reported to increase breast cancer susceptibility in African-American women, whereas the mitochondrial NADH dehydrogenase subunit 3 (ND3) polymorphism (A10398G) is linked to sporadic breast cancer in Poland (Canter et al., 2005; Czarnecka et al., 2010). It was also shown that mtDNA $10398 \mathrm{G}$ allele carriers have increased breast cancer risk due to alcohol consumption, perhaps as a result of the alcohol metabolite, acetaldehyde, which increases oxidative stress-induced damage of mtDNA (Pezzotti et al., 2009; Rohan et al., 2010). Smoking not only increases oxidative stress, but also augments mtDNA copy number, which correlates with an increased risk for breast cancer (Rohan et al., 2010; Shen et al., 2010).

It was reported that oxidative stress leads to HIF-1a accumulation and $j u n D$ gene inactivation in fibroblasts. This event likely activates fibroblasts that express elevated levels of stromal cell-derived factor (SDF1) and have expression profiles similar to carcinomaassociated fibroblast profiles (Toullec et al., 2010). Not surprisingly, accumulation of SDF1 in ErbB-2 positive breast cancer is linked with increased numbers of activated fibro-blasts and increased lymph node metastasis (Toullec et al., 2010). In addition, HIF-1a is known to regulate mitochondrial sirtuin NAD-dependent protein deacetylases 3 (SIRT3), a mitochondrial tumor suppressor, which maintains mitochondrial integrity and guards proper oxidative metabolism. Given the cancer preventive role of healthy mitochondria, it is not unexpected that loss of SIRT3 in fibroblasts would result in a tumorigenic phenotype and enhance breast cancer development (Kim et al., 2010). Healthy mitochondria are essential for maintaining energy, redox, and calcium balance. Impairments in mitochondrial function, and/or mechanisms controlling autophagy removal of damaged mitochondria lead to altered mitochondrial metabolisms and can promote cancer development. Another example of how oxidative stress may alter mitochondrial function to promote tumorigenesis through the microenvironment is via mammalian stanniocalcin 1 (STC1). STC1 has been found to be massively upregulated in mouse fibroblasts due to ROS accumulation (Nguyen, Chang, \& Reddel, 2009). Examination of STC-1 mRNA levels in multiple tumor samples showed that this glycoprotein was upregulated in many cancer types and might serve as a marker of micrometastasis (Fujiwara et al., 2000).

\section{CONCLUSIONS AND FUTURE PERSPECTIVES-SHOULD WE TARGET BREAST CANCER STROMA?}

Martinez-Outschoorn et al. described the tight connection between breast cancer cells and the stroma, using an MCF7-fibroblast co-culture system (Martinez-Outschoorn et al., 2011b). In this environment, the cancer cells produce hydrogen peroxide and by driving the "Reverse Warburg Effect" initiate oxidative stress in fibroblasts. As a result of this process, fibroblasts exhibited reduced mitochondrial activity, increased glucose uptake, ROS, and 
metabolite production. That obstructing oxidative stress in the tumor microenvironment can lead to mitophagy and promote breast cancer shutdown is a promising discovery for the development of future therapeutic interventions. In healthy organisms, hydrogen peroxide produced during the wound healing process by a normal epithelium is sufficient to convert normal fibroblasts to activated myofibroblasts that go on to produce hydrogen peroxide themselves, enhancing the inflammatory signal. Activation of the cancer microenvironment activation is constant, and CAFs never undergo nemosis; this is in contrast to the normal wound healing process (Kankuri et al., 2005, 2008). Nemosis is a type of nonapoptotic cell death characterized by a necrotic morphology in fibroblasts. Some investigators have promoted the idea of targeting CAFs, activated fibroblast, or fibroblasts in the senescence secretory phenotype state or the autophagy transition phase by cutting off all the "breast cancer fuel," L-lactate, ketone bodies, glutamine, and free fatty acids, thereby preventing breast cancer progression. Removing oxidative stress from the microenvironment by antioxidant treatment is a very tempting strategy. However, diets rich in antioxidants have fallen short in sufficiently preventing cancer. This clearly emphasizes the diverse roles of oxidative stress in a healthy organism and the need for a more detailed understanding of how oxidative stress specifically promotes cancer. It is clear that a healthy life style in combination with a healthy diet rich in fruits and vegetables, low in saturated fatty acids and free of alcohol and cigarette smoking is beneficial in supporting the prevention of breast cancer. Considerable data exists to support the premise that a lack of antioxidant enzymes tips the balance toward cancer initiation. For example, mice deficient in the peroxidase, Prdx1, die prematurely of oxidationinduced anemia and cancer (Neumann et al., 2003), suggesting a tumor preventive role for Prdx 1 . Fibroblasts lacking Prdx 1 are characterized by hyperactive Akt, DNA damage, elevated cellular ROS; Prdx-1 null mice expressing oncogenic H-Ras in the mammary gland have a higher incidence of breast cancer compared to wild-type mice expressing oncogenic (Cao et al., 2009; Neumann, Cao, \& Manevich, 2009). Prdx 1 is a safeguard of the PI3K/Akt signaling pathway as a result of its ability to protect the tumor suppressor PTEN from oxidation-induced inactivation. Knockdown PTEN in mouse mammary stromal fibroblasts increases microenvironment remodeling, collagen deposition, angiogenesis, immune cell recruitment, and increased malignancy of mammary epithelial tumor (Trimboli et al., 2009), supporting the premise that oxidative stress may promote breast cancer in part, by inactivating PTEN. Along these lines, drugs targeting oxidative mitochondrial oxidative phosphorylation have recently been developed for support of breast cancer treatment (Whitaker-Menezes et al., 2011a). Antioxidants like $N$-acetyl cysteine, hydroxy-chloroquine, and metformin prevent oxidative stress in CAFs and ketones, lactate, and glutamine from being transported into breast cancer cells (Sonveaux et al., 2008; Whitaker-Menezes et al., 2011b). In conclusion, regulating the crosstalk between tumor and stroma (Fig. 3.3) or specifically targeting CAFs, which are genetically more stable than tumor cells may provide a viable option in the future for both the prevention and treatment of breast cancer (Hiscox, Barrett-Lee, \& Nicholson, 2011; Loeffler et al., 2006). However, to pursue this goal successfully, additional research is needed to define the molecular details of how oxidative stress promotes cancer through the tumor microenvironment.

\section{REFERENCES}

Balliet RM, et al. Mitochondrial oxidative stress in cancer-associated fibroblasts drives lactate production, promoting breast cancer tumor growth: Understanding the aging and cancer connection. Cell Cycle. 2011; 10(23):4065-4073. [PubMed: 22129993]

Bardeesy N, et al. Both p16(Ink4a) and the p19(Arf )-p53 pathway constrain progression of pancreatic adenocarcinoma in the mouse. Proceedings of the National Academy of Sciences of the United States of America. 2006; 103(15):5947-5952. [PubMed: 16585505] 
Basuroy S, et al. Nox4 NADPH oxidase mediates oxidative stress and apoptosis caused by TNF-alpha in cerebral vascular endothelial cells. American Journal of Physiology. Cell Physiology. 2009; 296(3):C422-C432. [PubMed: 19118162]

Boidot R, et al. Regulation of monocarboxylate transporter MCT1 expression by p53 mediates inward and outward lactate fluxes in tumors. Cancer Research. 2012; 72(4):939-948. [PubMed: 22184616]

Campisi J. Aging, cellular senescence, and cancer. Annual Review of Physiology. 2013; 75:685-705.

Canter JA, et al. Mitochondrial DNA G10398A polymorphism and invasive breast cancer in AfricanAmerican women. Cancer Research. 2005; 65(17):8028-8033. [PubMed: 16140977]

Cao J, et al. Prdx 1 inhibits tumorigenesis via regulating PTEN/AKT activity. The EMBO Journal. 2009; 28(10):1505-1517. [PubMed: 19369943]

Capparelli C, et al. CDK inhibitors (p16/p19/p21) induce senescence and autophagy in cancerassociated fibroblasts, "fueling" tumor growth via paracrine interactions, without an increase in neoangiogenesis. Cell Cycle. 2012a; 11(19):3599-3610. [PubMed: 22935696]

Capparelli C, et al. Autophagy and senescence in cancer-associated fibroblasts metabolically supports tumor growth and metastasis via glycolysis and ketone production. Cell Cycle. 2012b; 11(12):22852302. [PubMed: 22684298]

Capparelli C, et al. CTGF drives autophagy, glycolysis and senescence in cancer-associated fibroblasts via HIF1 activation, metabolically promoting tumor growth. Cell Cycle. 2012c; 11(12):22722284. [PubMed: 22684333]

Chiavarina B, et al. HIF1-alpha functions as a tumor promoter in cancer associated fibroblasts, and as a tumor suppressor in breast cancer cells: Autophagy drives compartment-specific oncogenesis. Cell Cycle. 2010; 9(17):3534-3551. [PubMed: 20864819]

Comito $\mathrm{G}$, et al. Stromal fibroblasts synergize with hypoxic oxidative stress to enhance melanoma aggressiveness. Cancer Letters. 2012; 324(1):31-41. [PubMed: 22659468]

Coppe JP, et al. A human-like senescence-associated secretory phenotype is conserved in mouse cells dependent on physiological oxygen. PLoS One. 2010; 5(2):e9188. [PubMed: 20169192]

Czarnecka AM, et al. Mitochondrial NADH-dehydrogenase subunit 3 (ND3) polymorphism (A10398G) and sporadic breast cancer in Poland. Breast Cancer Research and Treatment. 2010; 121(2):511-518. [PubMed: 19266278]

Edderkaoui M, et al. NADPH oxidase activation in pancreatic cancer cells is mediated through Aktdependent up-regulation of p22phox. Journal of Biological Chemistry. 2011; 286(10):7779-7787. [PubMed: 21118808]

Felty Q. Redox sensitive Pyk2 as a target for therapeutics in breast cancer. Frontiers in Bioscience. $2011 ; 16: 568-577$.

$\mathrm{Fu}$ X, et al. Hypochlorous acid oxygenates the cysteine switch domain of promatrilysin (MMP-7). A mechanism for matrix metalloproteinase activation and atherosclerotic plaque rupture by myeloperoxidase. Journal of Biological Chemistry. 2001; 276(44):41279-41287. [PubMed: 11533038]

Fujiwara Y, et al. Assessment of Stanniocalcin-1 mRNA as a molecular marker for micrometastases of various human cancers. International Journal of Oncology. 2000; 16(4):799-804. [PubMed: 10717250]

Goh J, et al. Mitochondrial targeted catalase suppresses invasive breast cancer in mice. BMC Cancer. 2011; 11:191. [PubMed: 21605372]

Gonzalez-Angulo AM, et al. PI3K pathway mutations and PTEN levels in primary and metastatic breast cancer. Molecular Cancer Therapeutics. 2011; 10(6):1093-1101. [PubMed: 21490305]

Graham KA, et al. NADPH oxidase 4 is an oncoprotein localized to mitochondria. Cancer Biology \& Therapy. 2010; 10(3):223-231. [PubMed: 20523116]

Haviv I, et al. Origin of carcinoma associated fibroblasts. Cell Cycle. 2009; 8(4):589-595. [PubMed: 19182519]

Hecker L, et al. NADPH oxidase-4 mediates myofibroblast activation and fibrogenic responses to lung injury. Nature Medicine. 2009; 15(9):1077-1081.

Heering J, Erlmann P, Olayioye MA. Simultaneous loss of the DLC1 and PTEN tumor suppressors enhances breast cancer cell migration. Experimental Cell Research. 2009; 315(15):2505-2514. [PubMed: 19482022] 
Hiscox S, Barrett-Lee P, Nicholson RI. Therapeutic targeting of tumor-stroma interactions. Expert Opinion on Therapeutic Targets. 2011; 15(5):609-621. [PubMed: 21388336]

Hyoudou K, et al. SOD derivatives prevent metastatic tumor growth aggravated by tumor removal. Clinical \& Experimental Metastasis. 2008; 25(5):531-536. [PubMed: 18357506]

Jensen JD, et al. PIK3CA mutations, PTEN, and pHER2 expression and impact on outcome in HER2positive early-stage breast cancer patients treated with adjuvant chemotherapy and trastuzumab. Annals of Oncology. 2012; 23(8):2034-2042. [PubMed: 22172323]

Kalluri R, Zeisberg M. Fibroblasts in cancer. Nature Reviews. Cancer. 2006; 6(5):392-401.

Kankuri E, et al. Induction of hepatocyte growth factor/scatter factor by fibro-blast clustering directly promotes tumor cell invasiveness. Cancer Research. 2005; 65(21):9914-9922. [PubMed: 16267016]

Kankuri E, et al. Fibroblast nemosis arrests growth and induces differentiation of human leukemia cells. International Journal of Cancer. 2008; 122(6):1243-1252.

Kim HS, et al. SIRT3 is a mitochondria-localized tumor suppressor required for maintenance of mitochondrial integrity and metabolism during stress. Cancer Cell. 2010; 17(1):41-52. [PubMed: 20129246]

Koch S, et al. A new redox-dependent mechanism of MMP-1 activity control comprising reduced lowmolecular-weight thiols and oxidizing radicals. Journal of Molecular Medicine (Berlin). 2009; 87(3):261-272.

Koppenol WH, Bounds PL, Dang CV. Otto Warburg's contributions to current concepts of cancer metabolism. Nature Reviews. Cancer. 2011; 11(5):325-337.

Krtolica A, et al. Senescent fibroblasts promote epithelial cell growth and tumori-genesis: A link between cancer and aging. Proceedings of the National Academy of Sciences of the United States of America. 2001; 98(21):12072-12077. [PubMed: 11593017]

Lin $\mathrm{CH}$, Lin PH. Induction of ROS formation, poly(ADP-ribose) polymerase-1 activation, and cell death by PCB126 and PCB153 in human T47D and MDA-MB-231 breast cancer cells. ChemicoBiological Interactions. 2006; 162(2):181-194. [PubMed: 16884709]

Lisanti MP, et al. Characterization of caveolin-rich membrane domains isolated from an endothelialrich source: Implications for human disease. The Journal of Cell Biology. 1994; 126(1):111-126. [PubMed: 7517942]

Loeffler M, et al. Targeting tumor-associated fibroblasts improves cancer chemotherapy by increasing intratumoral drug uptake. The Journal of Clinical Investigation. 2006; 116(7):1955-1962. [PubMed: 16794736]

Martinez-Outschoorn UE, et al. Tumor cells induce the cancer associated fibro-blast phenotype via caveolin-1 degradation: Implications for breast cancer and DCIS therapy with autophagy inhibitors. Cell Cycle. 2010a; 9(12):2423-2433. [PubMed: 20562526]

Martinez-Outschoorn UE, et al. Autophagy in cancer associated fibroblasts promotes tumor cell survival: Role of hypoxia, HIF1 induction and NFkappaB activation in the tumor stromal microenvironment. Cell Cycle. 2010b; 9(17):3515-3533. [PubMed: 20855962]

Martinez-Outschoorn UE, et al. Oxidative stress in cancer associated fibroblasts drives tumor-stroma co-evolution: A new paradigm for understanding tumor metabolism, the field effect and genomic instability in cancer cells. Cell Cycle. 2010c; 9(16):3256-3276. [PubMed: 20814239]

Martinez-Outschoorn UE, et al. Anti-estrogen resistance in breast cancer is induced by the tumor microenvironment and can be overcome by inhibiting mitochondrial function in epithelial cancer cells. Cancer Biology \& Therapy. 2011a; 12(10):924-938. [PubMed: 22041887]

Martinez-Outschoorn UE, et al. Cancer cells metabolically "fertilize" the tumor microenvironment with hydrogen peroxide, driving the Warburg effect: Implications for PET imaging of human tumors. Cell Cycle. 2011b; 10(15):2504-2520. [PubMed: 21778829]

Martinez-Outschoorn UE, et al. Understanding the metabolic basis of drug resistance: Therapeutic induction of the Warburg effect kills cancer cells. Cell Cycle. 2013; 10(15):2521-2528. [PubMed: 21768775]

Marumo T, et al. Platelet-derived growth factor-stimulated superoxide anion production modulates activation of transcription factor NF-kappaB and expression of monocyte chemoattractant protein 1 in human aortic smooth muscle cells. Circulation. 1997; 96(7):2361-2367. [PubMed: 9337212] 
Meng D, Lv DD, Fang J. Insulin-like growth factor-I induces reactive oxygen species production and cell migration through Nox4 and Rac1 in vascular smooth muscle cells. Cardiovascular Research. 2008; 80(2):299-308. [PubMed: 18567639]

Neumann CA, Cao J, Manevich Y. Peroxiredoxin 1 and its role in cell signaling. Cell Cycle. 2009; 8(24):4072-4078. [PubMed: 19923889]

Neumann CA, et al. Essential role for the peroxiredoxin Prdx 1 in erythrocyte anti-oxidant defence and tumour suppression. Nature. 2003; 424(6948):561-565. [PubMed: 12891360]

Nguyen A, Chang AC, Reddel RR. Stanniocalcin-1 acts in a negative feedback loop in the prosurvival ERK1/2 signaling pathway during oxidative stress. Oncogene. 2009; 28(18):1982-1992. [PubMed: 19347030]

Nishikawa M, et al. Inhibition of metastatic tumor growth by targeted delivery of antioxidant enzymes. 109. Journal of Controlled Release. 2005; (1-3):101-107.

Noh EM, et al. Estrogen receptor alpha induces down-regulation of PTEN through PI3-kinase activation in breast cancer cells. Molecular Medicine Reports. 2011; 4(2):215-219. [PubMed: 21468554]

Parrinello S, et al. Stromal-epithelial interactions in aging and cancer: Senescent fibroblasts alter epithelial cell differentiation. Journal of Cell Science. 2005; 118(Pt 3):485-496. [PubMed: 15657080]

Pavlides S, et al. The reverse Warburg effect: Aerobic glycolysis in cancer associated fibroblasts and the tumor stroma. Cell Cycle. 2009; 8(23):3984-4001. [PubMed: 19923890]

Pezzotti A, et al. The mitochondrial A10398G polymorphism, interaction with alcohol consumption, and breast cancer risk. PLoS One. 2009; 4(4):e5356. [PubMed: 19390621]

Provenzano PP, et al. Collagen density promotes mammary tumor initiation and progression. BMC Medicine. 2008; 6:11. [PubMed: 18442412]

Radisky DC, et al. Rac1b and reactive oxygen species mediate MMP-3-induced EMT and genomic instability. Nature. 2005; 436(7047):123-127. [PubMed: 16001073]

Rohan TE, et al. Do alterations in mitochondrial DNA play a role in breast carcinogenesis? Journal of Oncology. 2010; 2010:604304. [PubMed: 20628528]

Russillo M, et al. Assessment of PTEN and PI3K status in primary breast cancer and corresponding metastases: Is it worthwhile? Journal of Clinical Oncology. 2011; 29(20):2834-2835. author reply 2835. [PubMed: 21670454]

Sampson N, et al. ROS signaling by NOX4 drives fibroblast-to-myofibroblast differentiation in the diseased prostatic stroma. Molecular Endocrinology. 2011; 25(3):503-515. [PubMed: 21273445]

Sappino AP, et al. Smooth-muscle differentiation in stromal cells of malignant and non-malignant breast tissues. International Journal of Cancer. 1988; 41(5):707-712.

Sharpless NE, et al. The differential impact of p16(INK4a) or p19(ARF) deficiency on cell growth and tumorigenesis. Oncogene. 2004; 23(2):379-385. [PubMed: 14724566]

Shen J, et al. Mitochondrial copy number and risk of breast cancer: A pilot study. Mitochondrion. 2010; 10(1):62-68. [PubMed: 19788937]

Siegel R, Naishadham D, Jemal A. Cancer statistics, 2012. CA: A Cancer Journal for Clinicians. 2012; 62(1):10-29. [PubMed: 22237781]

Sonveaux P, et al. Targeting lactate-fueled respiration selectively kills hypoxic tumor cells in mice. The Journal of Clinical Investigation. 2008; 118(12):3930-3942. [PubMed: 19033663]

Sotgia F, Martinez-Outschoorn UE, Lisanti MP. Mitochondrial oxidative stress drives tumor progression and metastasis: Should we use antioxidants as a key component of cancer treatment and prevention? BMC Medicine. 2011; 9:62. [PubMed: 21605374]

Sotgia F, et al. Understanding the Warburg effect and the prognostic value of stromal caveolin-1 as a marker of a lethal tumor microenvironment. Breast Cancer Research. 2011; 13(4):213. [PubMed: 21867571]

Sotgia F, et al. Caveolin-1 and cancer metabolism in the tumor microenvironment: Markers, models, and mechanisms. Annual Review of Pathology. 2012; 7:423-467. 
Stolarek RA, et al. Increased $\mathrm{H}_{2} \mathrm{O}_{2}$ level in exhaled breath condensate in primary breast cancer patients. Journal of Cancer Research and Clinical Oncology. 2010; 136(6):923-930. [PubMed: 19967414]

Suda T, et al. ER-activating ability of breast cancer stromal fibroblasts is regulated independently of alteration of TP53 and PTEN tumor suppressor genes. Biochemical and Biophysical Research Communications. 2012; 428(2):259-263. [PubMed: 23068099]

Taddei ML, et al. Mitochondrial oxidative stress due to complex I dysfunction promotes fibroblast activation and melanoma cell invasiveness. Journal of Signal Transduction. 2012:684592. [PubMed: 22272371]

Tanic N, et al. The impact of PTEN tumor suppressor gene on acquiring resistance to tamoxifen treatment in breast cancer patients. Cancer Biology \& Therapy. 2012; 13(12):1165-1174. [PubMed: 22892847]

Tobar N, et al. NOX4-dependent ROS production by stromal mammary cells modulates epithelial MCF-7 cell migration. British Journal of Cancer. 2010; 103(7):1040-1047. [PubMed: 20717118]

Toullec A, et al. Oxidative stress promotes myofibroblast differentiation and tumour spreading. EMBO Molecular Medicine. 2010; 2(6):211-230. [PubMed: 20535745]

Trimboli AJ, et al. Pten in stromal fibroblasts suppresses mammary epithelial tumours. Nature. 2009; 461(7267):1084-1091. [PubMed: 19847259]

Trimmer C, et al. Caveolin-1 and mitochondrial SOD2 (MnSOD) function as tumor suppressors in the stromal microenvironment: A new genetically tractable model for human cancer associated fibroblasts. Cancer Biology \& Therapy. 2011; 11(4):383-394. [PubMed: 21150282]

Waghray M, et al. Hydrogen peroxide is a diffusible paracrine signal for the induction of epithelial cell death by activated myofibroblasts. The FASEB Journal. 2005; 19(7):854-856.

Weitzman JB. p16(Ink4a) and p19(Arf ): Terrible twins. Trends in Molecular Medicine. 2001; 7(11): 489. [PubMed: 11689318]

Whitaker-Menezes D, et al. Hyperactivation of oxidative mitochondrial metabolism in epithelial cancer cells in situ: Visualizing the therapeutic effects of metformin in tumor tissue. Cell Cycle. 2011a; 10(23):4047-4064. [PubMed: 22134189]

Whitaker-Menezes D, et al. Evidence for a stromal-epithelial "lactate shuttle" in human tumors: MCT4 is a marker of oxidative stress in cancer-associated fibroblasts. Cell Cycle. 2011b; 10(11):17721783. [PubMed: 21558814]

Witkiewicz AK, et al. Loss of stromal caveolin-1 expression predicts poor clinical outcome in triple negative and basal-like breast cancers. Cancer Biology \& Therapy. 2010; 10(2):135-143. [PubMed: 20431349]

Witkiewicz AK, et al. Molecular profiling of a lethal tumor microenvironment, as defined by stromal caveolin-1 status in breast cancers. Cell Cycle. 2011; 10(11):1794-1809. [PubMed: 21521946]

Witkiewicz AK, et al. Using the "reverse Warburg effect" to identify high-risk breast cancer patients: stromal MCT4 predicts poor clinical outcome in triple-negative breast cancers. Cell Cycle. 2012; 11(6):1108-1117. [PubMed: 22313602] 


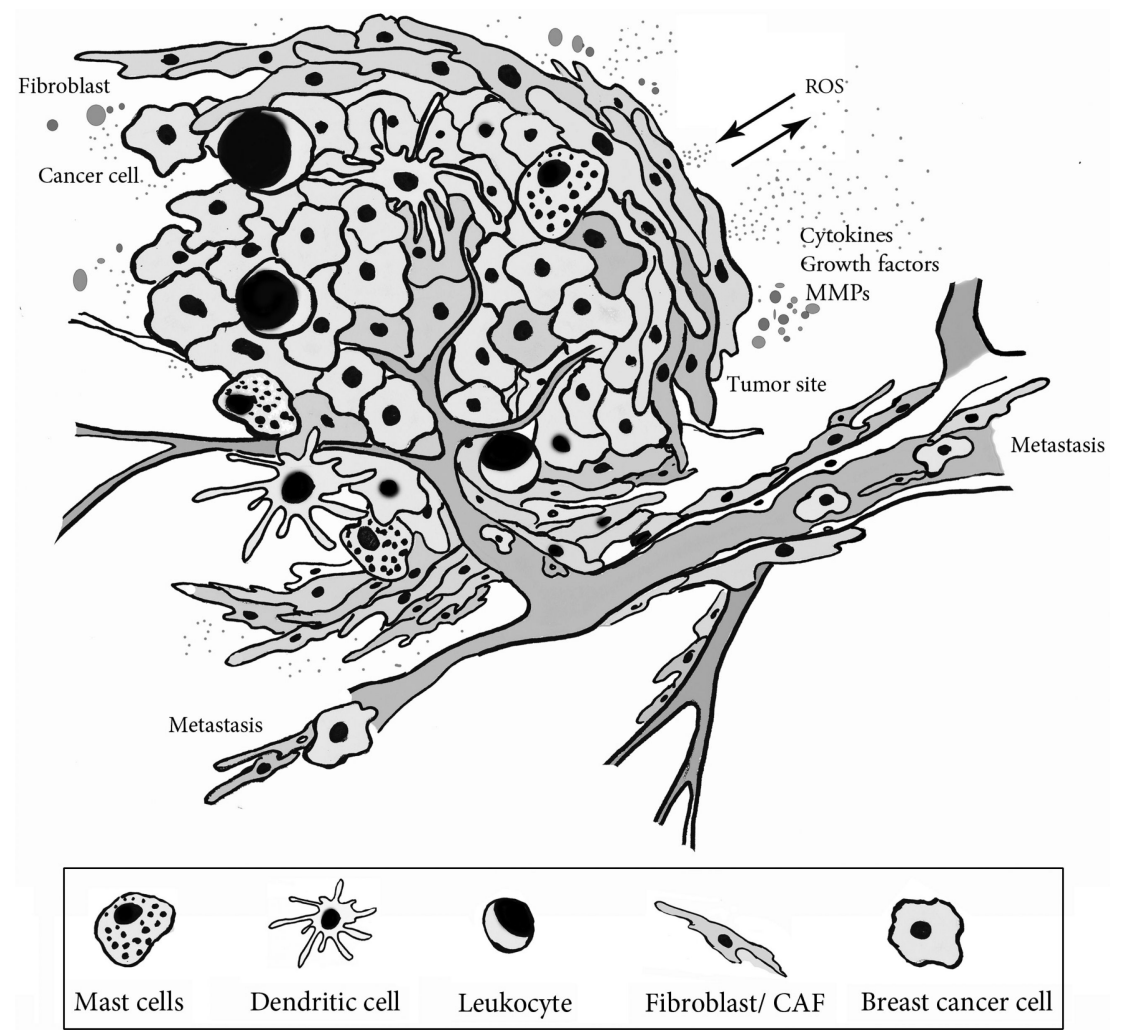

Figure 3.1.

Breast tumor microenvironment. The tumor microenvironment consists of cancer, stromal, and nonmalignant cells supporting tumor growth and vascularization. Under oxidative stress, activated stromal cells generate tumor enhancing signals. Activated fibroblasts acquire a contractile phenotype and mobility affecting cancer cell migration and invasion. Immune cells infiltrate the tumor tissue and secrete cytokines supporting further microenvironmental stress. (See Page 1 in Color Section at the back of the book.) 


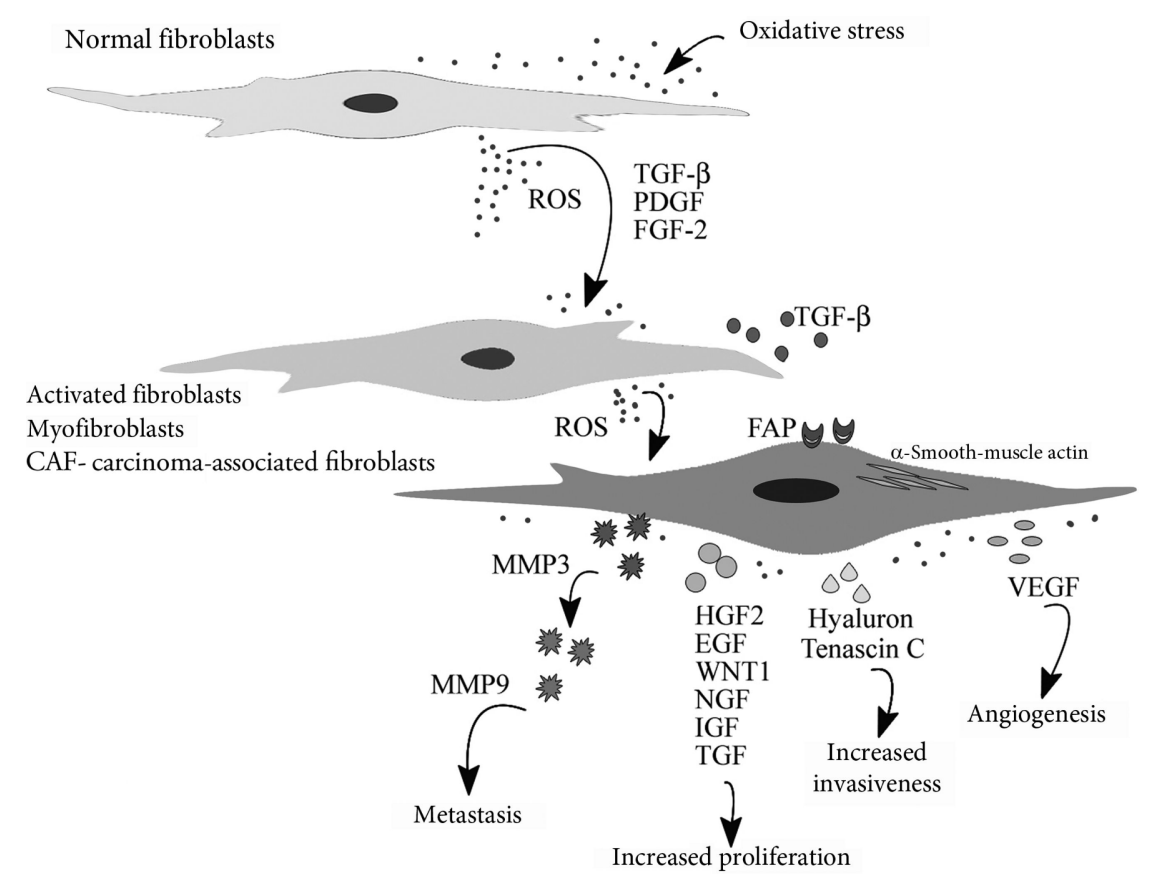

Figure 3.2.

Oxidative stress activates stroma fibroblasts. In breast cancer, under oxidative stress, stroma-associated fibroblasts acquire an activated state and can be identified by numerous markers including a-smooth muscle actin (a-SMA), fibroblast specific protein (FSP), fibroblast activation protein (FAP), PDGF receptors- $\beta$, desmin, endosialin, tenascin-c, palladin, vimentin, pro-collagen, stromelysin-3, and cadherin-11. Oxidative stress can cause cell senescence, locking activated fibroblast in a nonproliferative state and creating a senescence-associated secretory phenotype, which manifests in releasing factors that affect neighboring cells. Activation of fibroblasts, followed by secretion of numerous factors including metalloproteinases (MMP-2, MMP-3, MMP9), growth factors (HGF-2, EGF, IGF, TGF $\beta$, VEGF), the polysaccharide hyaluronan and tenascin-c significantly promotes proliferation and growth of premalignant epithelial cells and cancer cells, invasiveness, metastasis, and angiogenesis in breast cancer. (See Page 2 in Color Section at the back of the book.) 


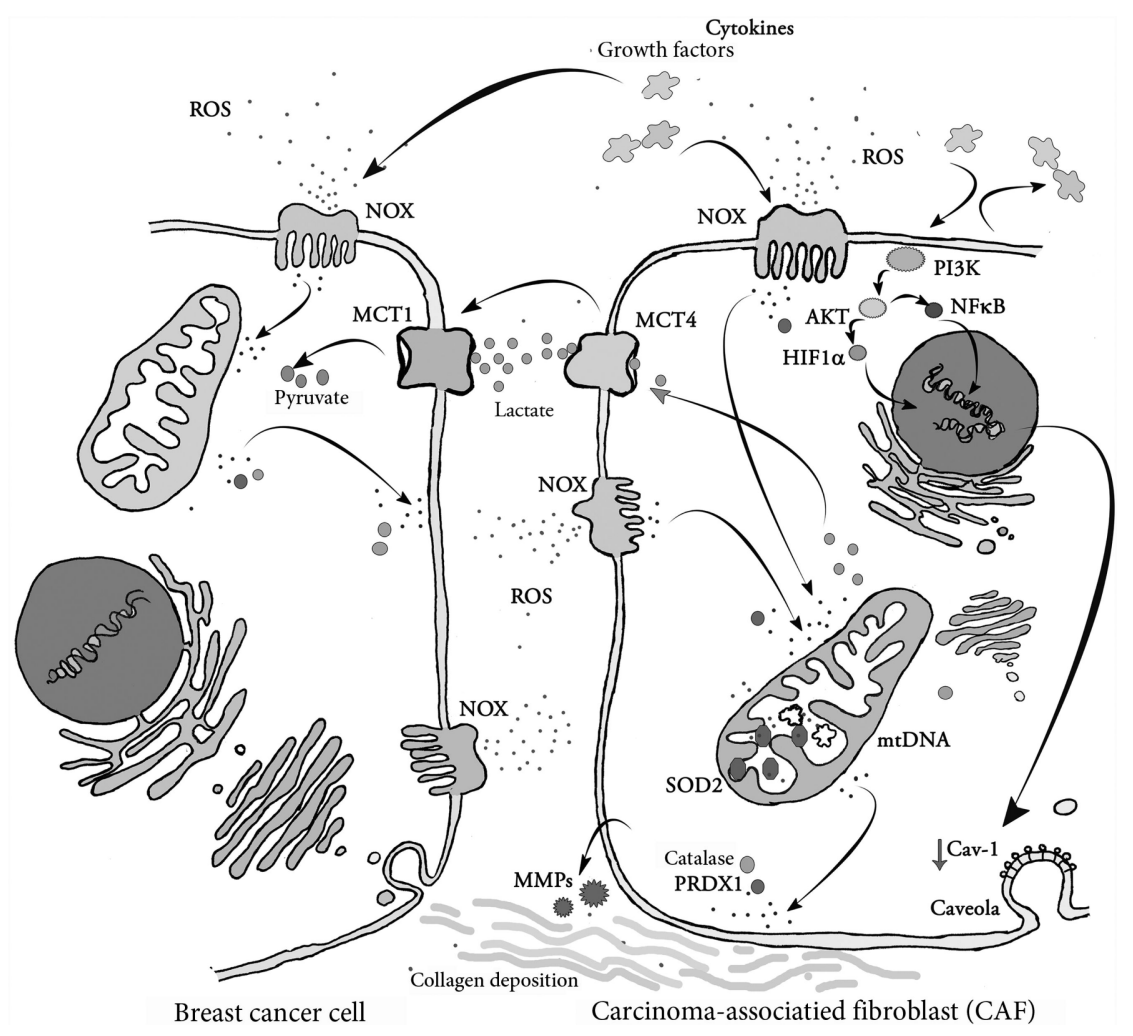

Figure 3.3.

Interactions between CAFs and breast cancer cells in the tumor microenvironment. In the tumor microenvironment close interactive pathways are established, cross-linking breast cancer cells and stromal cells. Upregulated NADPH oxidase complex (NOX) bound in cell membranes is a source of large amounts of ROS released from these cells. Cancer cells secrete ROS, cytokines, and growth factors, prompting a reactive response in the stroma. Activated stromal cells influence cancer cell malignancy by increasing oxidative stress, collagen deposition, MMP, and growth factor secretion. Oxidative stress-induced loss of expression of membrane-bound scaffolding protein, caveolin-1 (Cav-1), in cancer-associated fibroblasts via autophagy also results in an activated tumor microenvironment. The uncontrolled expansion of cancer cells depends upon high ATP production and high glucose availability. CAFs release lactate via monocarboxylate transporter 4 (MCT4) and monocarboxylate transporter 1 (MCT1) on cancer cells facilitating lactate uptake. Thus, by providing high energy metabolites activated fibroblasts maintain continuous cancer progression. (See Page 3 in Color Section at the back of the book.) 